invited by reception staff to leave the clinic until they were sent another text when they were due to be seen. Patients in possession of a Smartphone could refresh a link to check their place in the queue at any time. IR1s and patient feedback were assessed before and after implementation

Results Average no of symptomatic patients seen over a weekend was 70 with an average wait time of 89 min. In the 4 month period prior to the software implementation there were 6 IR1 forms received from staff about patient aggression. In the 4 month period after its introduction there were none. Two months post its introduction the average number of patient complaints about waiting times received was 1 from an average of 4 prior to its use.

Conclusion The introduction of the queuing software has been an inexpensive and effective method of reducing complaints about patient waiting times and improving patient satisfaction with the service.

\section{CAN EXPRESS TREATMENT REDUCE ONWARD TRANSMISSION?}

Ruth Byrne*, Farhad Cooper, Tim Appleby, Leigh Chislett, Lucy Freeman, Elizabeth Kershaw, Nneka Nwokolo, Gary Whitlock, Alan Mcowan. Chelsea and Westminster Hospital, London, UK

\subsection{6/sextrans-2015-052126.19}

Background/introduction The introduction of onsite Cepheid ${ }^{\circledR}$ GeneXpert diagnostics for asymptomatic STI screens cut 'test to treatment' time by $190 \mathrm{~h}$.

Aim(s)/objectives To evaluate the Public Health benefit of faster treatment.

Methods Patients with chlamydia (CT) and/or gonorrhoea (GC) over 8 weeks in February 2014 were retrospectively identified. We compared the timing of testing, treatment and number of recent sexual partners with a control group from November 2013. Assuming rate of partners remains unchanged, we calculated 'partners spared' exposure per infected patient due to faster treatment.

Results 431 patients were identified with CT and/or GC infection. 81\% (349/431) were MSM. Median age was 29 years. $23 \%$ of index patients disclosed high risk behaviour including fisting, chemsex and injecting drug use. Median 'test to treatment' time dropped from $238 \mathrm{~h}$ to $48 \mathrm{~h}$. The number of partners spared exposure was 0.5 per index case. This equates to a total 196 partners spared exposure over the study period.

Discussion/conclusion For every two people diagnosed with an infection, one partner was spared exposure. Limiting the duration of infectivity and the potential for onward transmission has clear public health benefits and is of particular value in this cohort with multiple partners who engage in high-risk behaviour.

\section{O20 ON-LINE STI TESTING SERVICES: IMPROVING ACCESS, EFFICIENCY AND USER EXPERIENCE}

${ }^{1}$ Michael Brady ${ }^{*},{ }^{2}$ Chris Howroyd, ${ }^{2}$ Glyn Parry, ${ }^{1,2}$ Paula Baraitser, ${ }^{2,3}$ Gillian Holdsworth, ${ }^{4}$ Anatole Menon-Johansson. 'Kings College Hospital NHS Foundation Trust, London, Norway; ${ }^{2}$ SH:24, London, UK; ${ }^{3}$ Lambeth and Southwark Public Health Department, London, UK; ${ }^{4}$ Guys and St Thomas' NHS Foundation Trust, London, UK

10.1136/sextrans-2015-052126.20
Background/introduction There are many barriers to accessing sexual health and HIV testing services. Novel service models could address this. On-line testing may provide a solution.

Aim(s)/objectives To evaluate the acceptability and potential impact of on-line STI testing.

Methods We developed a dedicated, secure website for free online STI testing. Website content and testing process was iteratively designed in response to user feedback. Simple questions identify those most at risk or symptomatic and signpost to local services. Clients order self-taken NAAT tests for chlamydia (CT) and gonorrhoea (GC) and a pin-prick blood test for syphilis and $4^{\text {th }}$ generation HIV testing and post them to the laboratory. Results are received by text. In November 2014 we piloted the process by offering it to clients attending 2 sexual health services.

Results 47 clients used the service. 31 (65.9\%) men, of whom 5 (16\%) were MSM. Mean age was 29 (range 19-64). Mean time to receipt of results was 3 days (range 0-8). 18 (38.3\%) clients received their results on the same day the sample was taken. One client tested positive for syphilis. All other tests were negative. User feedback was predominantly positive, with specific reference to its speed and simplicity. $8 / 47$ (17\%) left negative feedback about the pinprick process, which they found difficult or unpleasant.

Discussion/conclusion The service was highly acceptable. Rapid results turnaround was more efficient than local 'traditional' services. The service (which soon becomes available to all local residents) will contribute significantly to local STI/HIV testing and prevention strategies.

\section{SECURING EXCELLENCE IN CHLAMYDIA SCREENING OUTCOMES ON A SHRINKING BUDGET}

${ }^{1}$ Sharon Foster, ${ }^{1}$ Victoria Womack, ${ }^{2}$ Sharron Ainslie, ${ }^{2}$ Kate A Folkard, ${ }^{2}$ Kevin Dunbar, ${ }^{2}$ John Saunders*. 'Leeds City Council, Leeds, UK; ${ }^{2}$ Public Health England, London, UK

\subsection{6/sextrans-2015-052126.21}

Background/introduction The National Chlamydia Screening Programme (NCSP) recommends opportunistically screening sexually active 15 to 24 year olds annually and on change of partner. Through a number of changes to the delivery of screening, Leeds has maintained a higher than average detection rate indicator (DRI) despite declining spend. We describe these changes and corresponding DRIs.

Aim(s)/objectives To review and re-structure chlamydia control activity to provide greater value for money.

Methods A multi-professional steering group was established and a strategic approach taken to commission chlamydia within sexual health services. Our approach included: screening, treatment and partner notification embedded within contraception and sexual health services; commissioning of online testing and an enhanced pharmacy scheme; signposting website developed; phasing out financial incentives for General Practitioners (GP); reducing outreach testing, marketing and staff.

Results In $2014 £ 371 \mathrm{k}$ was spent on screening activities $(£ 538 \mathrm{k}$ 2010/11). 2014 Q1-Q2 DRI was 3,104 (2,168 England; 2,325 Yorkshire and Humber) and 2,511 (1,888 England; 2,128 Y\&H), respectively compared to 2,698 (2,093 England; 2,367 $\mathrm{Y} \& \mathrm{H})$ and 2,355 (1,947 England; 2,068 Y\&H) for equivalent time periods in 2013. In 2013 most tests were performed in GP (30\%) followed by GUM (26.6\%), Internet (26.8\%) and CASH (13.5\%). Positivity across all settings in 2013 was $9.5 \%$. 
Discussion/conclusion By concentrating activity in venues with higher positivity, in line with guidance from the NCSP, it has been possible to achieve the DRI target whilst working within tighter economic constraints. In particular, outreach screening was costly and produced low volumes of tests with low positivity.

\section{O22 PERFORMANCE OF THE BD MAXTM CT/GC/TV ASSAY FOR DETECTION OF CHLAMYDIA, GONORRHOEA AND TRICHOMONAS}

${ }^{1}$ Barbara Van Der Pol*, ${ }^{1}$ Grace Daniel, ${ }^{2}$ James Williams, ${ }^{2}$ DeAnna Fuller, ${ }^{2}$ Thomas Davis, ${ }^{3}$ Stephanie Taylor, 'Edward Hook. 'University of Alabama at Birmingham, Birmingham, AL, USA; ${ }^{2}$ Indiana University School of Medicine, Indianapolis, IN, USA; ${ }^{3}$ Louisiana State University Health Sciences Center, New Orleans, LA, USA

\subsection{6/sextrans-2015-052126.22}

Background Chlamydia, gonorrhoea and trichomonas infections remain highly prevalent with annual WHO estimates of 106, 107 and 276 million cases respectively. Screening for all 3 infections in a single assay could improve control efforts.

Aim This study assessed the performance of the BD MAX ${ }^{\mathrm{TM}} \mathrm{CT} /$ GC/TV (BD MAX) for detection of chlamydia, gonorrhoea and trichomonas DNA compared to routine diagnostic methods.

Methods Urine, patient-collected vaginal and endocervical specimens were obtained from 1854 women. BD MAX assay results were compared to TV culture (InPouch), TV wet mount, Aptima $\mathrm{AC} 2$ and TV assays and the BD Viper ${ }^{\mathrm{TM}} \mathrm{CTQ}^{\mathrm{x}} / \mathrm{GCQ}^{\mathrm{x}}$ assays.

Results Prevalence for chlamydia, gonorrhoea and trichomonas was $7.3,2.3$ and $14.7 \%$, respectively. Sensitivity estimates ranged from 92.2-99.2, 94.9-95.1 and 92.9-96.1 for chlamydia, gonorrhoea and trichomonas, respectively. Specificity estimates for each test were $\geq 98.6$. Of the 128 out of 1758 (7.3\%) women with chlamydia infections, concomitant gonococcal and trichomonal infections were present in 11.7 and $12.5 \%$, respectively. The sensitivity of the assay for chlamydia when co-infections were present ranged from 92.6-96.1\%. Similarly the sensitivity of the gonorrhoea and trichomonas detection was not affected by the presence of concomitant chlamydial infections with estimates ranging from $93.8-100 \%$ and $89.5-100 \%$, respectively.

Discussion The performance of the BD MAX assay was similar to that of other molecular diagnostic assays. A substantial proportion of women with chlamydia are co-infected with gonorrhoea and/or trichomonas. Trichomonas was more prevalent than chlamydia and gonorrhoea combined. Detection of all three infections in a single assay may improve identification and treatment of these STI.

\section{O23 PORA PSEUDOGENE DELETION AMONGST NEISSERIA GONORRHOEAE ISOLATES FROM THE GONOCOCCAL RESISTANCE TO ANTIMICROBIALS SURVEILLANCE PROGRAMME (GRASP)}

${ }^{1}$ Martina Toby, ${ }^{2}$ Pamela Saunders, ${ }^{2}$ Michelle Cole, ${ }^{2}$ Vlad Grigorjev, ${ }^{2}$ Sarah Alexander*, ${ }^{2}$ Cathy Ison. 'Guys and St Thomas' NHS Foundation Trust, London, UK; ${ }^{2}$ Public Health England, London, UK

\subsection{6/sextrans-2015-052126.23}

Background/introduction In the last four years, isolates of N. gonorrhoeae have been identified in Australia, Sweden,
Scotland and England which lack the gonococcal porA pseudogene and consequently result in negative results in the diagnostic porA pseudogene real-time-PCR (RT-PCR) for N. gonorrhoeae.

Aim(s)/objectives This study sought to determine the prevalence of porA pseudogene negative isolates amongst isolates received at Public Health England (PHE) through the national gonococcal resistance to antimicrobials surveillance programme (GRASP).

Methods DNA lysates were prepared from 533 N. gonorrhoeae isolates received from 20 centres via GRASP during 2011. Any isolate with a RT-PCR por A pseudogene negative result was repeated from a fresh culture and the porA gene was additionally DNA sequenced. Isolates were additionally tested using the gonococcal opa gene RT-PCR.

Results Four isolates $(4 / 533,0.8 \%)$ were found to be reproducibly negative with the porA pseudogene RT-PCR, but were positive with opa gene RT-PCR. DNA sequencing determined that two isolates contained the Neisseria meningitidis porA gene. Both isolates were from patients attending a clinic in SouthLondon.

Discussion/conclusion Less than one percent of the GRASP isolates from patients attending clinics across England expressed the meningococcal porA gene and therefore tested negative on the in-house porA assay. The low prevalence indicates that these isolates do not present a major diagnostic or public health problem. However, microbiologists should remain vigilant for any isolates giving anomalous results and when using the porA pseudogene RT-PCR consider multiplexing it with the opa-gene RT-PCR.

\section{O24 CONFIRMING GC NAAT RESULTS: IS IT ALWAYS NECESSARY?}

Sarah Alexander, Hemanti Patel, Simon Warwick, Helen Fifer*. Public Health England, London, UK

\subsection{6/sextrans-2015-052126.24}

Introduction Current guidance recommends that all specimens testing positive using a N. gonorrhoeae Nucleic Acid Amplification Test (GC NAAT) be confirmed using a second test with an alternative target, in order to achieve a positive predictive value above $90 \%$.

Aim To determine rates of GC NAAT confirmations by primary screening test and specimen site.

Methods 994 specimens which were GC NAAT positive at local laboratories were sent for confirmation using an in-house multiplex PCR with PorA and opa gene targets. A correlation between the confirmatory real-time PCR results, specimen site and GC screening NAAT was undertaken. For the purposes of this analysis equivocal results were regarded as positive and inhibited results were excluded.

Results Overall, $57 \%$ of specimens examined could be confirmed as GC positive using the in-house real-time PCR test (Table 1).

Discussion High rates of confirmation can be achieved when examining genital, rectal and urine specimens irrespective of the GC screening NAAT. However $>90 \%$ confirmatory rates were only achieved when examining male urine specimens which had been screened using the Probetec and Cobas Amplicor tests, 